\title{
Efeitos de um programa preventivo sobre desempenho funcional e controle postural em atletas de basquete
}

\author{
Effect of injury prevention program on functional performance and postural balance in \\ basketball players
}

Eduardo Nogueira Peres ${ }^{1}$

Simone Lara ${ }^{2}$

Lilian Pinto Teixeira ${ }^{3}$

Antonio Adolfo Mattos de Castro ${ }^{4}$

Ariel Aline Jardim Alves Escobar ${ }^{5}$

Endereço para correspondência:

Simone Lara

Universidade Federal do Pampa-

UNIPAMPA

Campus Uruguaiana/RS, BR 472, KM

592- CX Postal 118

97508-000, Uruguaiana, Rio Grande do

Sul, Brasil.

simonelara@unipampa.edu.br

\begin{abstract}
Resumo
Objetivo: avaliar os efeitos de um programa preventivo sobre a função muscular de extensores e flexores do joelho e o controle postural de atletas jovens de basquete.

Métodos: Esse estudo incluiu oito atletas de basquetebol (15 $\pm 1,06$ anos), no qual foram submetidos a uma avaliação da força muscular dos músculos da articulação do joelho, realizada através de um dinamômetro isocinético, e a uma avaliação do controle postural, por meio da posturografia dinâmica computadorizada. O programa de prevenção de lesão baseou-se em exercícios do programa FIFA11+, em exercícios funcionais e em exercícios de fortalecimento de quadril, realizados durante 12 semanas.

Resultados: Houve aumento do pico de torque (PT) e da potência (POT) de extensores e flexores do lado não-dominante no modo concêntrico, bem como da POT de flexores no lado dominante (modo concêntrico e excêntrico) pós-intervenção. Houve melhora quanto ao controle postural, pós-intervenção.

Conclusões: Encontramos efeitos benéficos de um programa preventivo sobre as variáveis analisadas.
\end{abstract}

Departamento de Saúde, Curso de Fisioterapia, Universidade Federal do Pampa- UNIPAMPA. Uruguaiana. Rio Grande do Sul, RS- Brasil. enogueiraperes@gmail.com

${ }_{2}$ (iD) Fisioterapeuta, Mestre em Fisiologia humana, Doutora em Educação em Ciências: química da vida e saúde, Docente do curso de Fisioterapia da UNIPAMPA, Campus Uruguaiana/RS - Brasil.

simonelara@unipampa.edu.br

Fisioterapeuta do curso de Fisioterapia, UNIPAMPA, Campus Uruguaiana/RS - Brasil. lipt19@yahoo.com.br

4 (iD) Fisioterapeuta, Mestre e Doutor em Ciências, Docente do curso de Fisioterapia da UNIPAMPA, Campus Uruguaiana/RS - Brasil.

antoniocastro@unipampa.edu.br

5 (iD) Fisioterapeuta, Pós-graduanda do Programa de Residência Multiprofissional em Saúde Coletiva, Universidade Federal do Pampa (UNIPAMPA), Campus Uruguaiana/RS - Brasil.

arielaline.escobar@gmail.com

Descritores: Basquetebol. Equilíbrio postural. Força muscular. Traumatismos em atletas.

\begin{abstract}
Objective: To evaluate the effect of Injury prevention program on knee extension/flexion muscle function and postural balance in young basketball players.

Methods: This study included eight basketball players ( $15 \pm 1.06$ years), that had their knee joint muscle strength assessed by means of a (1) isokinetic dynamometer and (2) their postural balance assessed by computadorized dynamic posturography. A warm-up programme to prevent injuries was based on FIFA11+, functional exercises and hip strengthening exercises, practiced during 12 weeks.

Results: We found increased muscle extensors and flexors torque peak (TP) and power (P) of the non dominant limb at concentric mode; as well, we found increased muscle flexor power of the dominant limb at concentric and eccentric modes after program completion. We found improvements in postural balance post intervention.

Conclusions: Our study showed that a prevention program improved investigated variables.
\end{abstract}

Keywords: Basketball. Postural balance. Muscle strength. Sport injuries.

\begin{abstract}
Cite como
Peres EN, Lara S, Teixeira LP, Castro AAM de, Escobar AAJA. Efeitos de um programa preventivo sobre desempenho funcional e controle postural em atletas de basquete Conscientiae Saúde 2019 jul./set.; 18(3):326-337. https://doi.org/10.5585/ConsSaude.v18n3 13691.
\end{abstract}




\section{Introdução}

O basquetebol é um esporte coletivo altamente dinâmico e de constante evolução técnica, tática e física ${ }^{1}$, apresentando características relacionadas à intensa performance, quando do correr, saltar e fintar. À essa atividade intensa, quando associado a um elevado cronograma de jogos, exige do atleta uma recuperação física de curto tempo ${ }^{2}$.

Conforme Moreira et al. ${ }^{3}$, a alta exigência física nos jogos e nos treinamentos intensos e extenuantes, demanda um alto esforço do atleta, predispondo ao desenvolvimento de lesões. Nesse sentido, um estudo recente ${ }^{4}$ evidenciou que, durante as temporadas de 2009/2010 e 2014/2015, da Associação Nacional de Basquete (NCAA), ocorreu um total de 2.308 lesões em atletas masculino, com taxas de lesão de $7,97 \%$ dos atletas a cada $1.000 \mathrm{~h}$ jogadas. Ainda nesse estudo, a maioria das lesões ocorreram nos membros inferiores desses atletas.

Com base nesses achados, a construção de estratégias de prevenção de lesões nessa modalidade esportiva é fundamental ${ }^{5}$. Assim, torna-se importante analisar variáveis que podem identificar fatores de risco para lesões, como a performance muscular e o controle postural.

A força muscular é uma variável comprovadamente importante para o desempenho humano na prática do basquetebol ${ }^{6,7}$, e, dentre as diversas formas de avaliação, o dinamômetro isocinético representa um método padrão ouro, que determina a performance funcional e o equilíbrio muscular ${ }^{8}$. Com ele, é possível avaliar valores absolutos, como os de torque, potência e trabalho de grupos musculares, além de valores relativos, como na relação de equilíbrio muscular agonista/antagonista, e quaisquer alterações podem identificar fatores de risco para lesões musculoesqueléticas ${ }^{9}$.

O controle postural é um processo complexo, que depende da integração da visão, da sensação vestibular e periférica, dos comandos centrais e de respostas neuromusculares e, particularmente, da força muscular e do tempo de reação, e a realização de gestos esportivos habilidosos dependem da integridade desse sistema ${ }^{10}$. De fato, no basquetebol, a realização de habilidades motoras complexas, como o salto, bandeja, rebotes e fintas requerem grande domínio sobre o equilíbrio postural ${ }^{11,12}$. Ainda, um sistema proprioceptivo alterado pode acarretar em déficits na estabilização articular e neuromuscular, que contribuem para a ocorrência de lesões ${ }^{13}$.

Atualmente, existem poucos estudos nacionais demonstrando efeitos de programas de prevenção de lesão em jogadores de basquetebol ${ }^{14}$. Com base nisso, o objetivo desse estudo foi 
avaliar os efeitos de um programa preventivo de 12 semanas sobre a função muscular de extensores e flexores do joelho e o controle postural em atletas jovens de basquete. Hipotetizamos que um programa preventivo de 12 semanas melhora a função muscular de extensores e flexores do joelho e o controle postural em atletas jovens de basquete.

\section{Material e métodos}

\section{Amostra}

Esse estudo prospectivo incluiu uma amostra de conveniência, formada por atletas de basquetebol, e, portanto, não houve a realização de cálculo amostral. O trabalho foi aprovado pelo Comitê de Ética em Pesquisa institucional ( $\mathrm{n}^{\circ}$ 2.036.667), e os responsáveis legais pelos sujeitos assinaram um Termo de Consentimento Livre e Esclarecido (TCLE). Os critérios de inclusão foram: jogadores do sexo masculino, com idades entre 14 a 17 anos, integrantes do time em treinamento regular, com tempo mínimo de 03 meses de treinamento. Os critérios de exclusão foram: atletas em afastamento do time por qualquer motivo de saúde ou em fase de reabilitação, e que não apresentassem frequência mínima de $75 \%$ no estudo.

A fim de caracterizar a amostra, foi avaliado a idade, a dominância dos membros inferiores (determinada através do relato do atleta) e dados antropométricos (massa e estatura), e os dados são apresentados na tabela 1 .

Tabela 1 - Caracterização da amostra

\begin{tabular}{ll}
\hline Variável & Média e DP \pm \\
\hline $\mathbf{N}$ & 8 \\
Idade (anos) & $15 \pm 1,06$ \\
Massa $(\mathrm{Kg})$ & $70,57 \pm 13,69$ \\
Estatura $(\mathrm{m})$ & $1,78 \pm 0,05$ \\
\hline
\end{tabular}

Dados descritivos, média e desvio padrão $\mathrm{DP} \pm$

\section{Instrumentos}

\section{Avaliação isocinética}

A performance muscular foi avaliada com o dinamômetro isocinético Biodex System Pro 4 (Biodex Medical Systems, Inc, Nova Iorque, EUA), sendo considerada uma ferramenta de pesquisa padrão ouro para a avaliação da função de grupos musculares ${ }^{8}$. A avaliação foi 
realizada nos modos concêntrico e excêntrico, no qual os sujeitos realizaram cinco repetições máximas de flexão e extensão do joelho, de ambas as pernas, com intervalo de um minuto entre as séries, avaliadas alternadamente nas velocidades de $60^{\circ}$.s.

Antes da realização do teste, os atletas realizaram um aquecimento de cinco minutos em uma bicicleta ergométrica sem carga, e executaram três repetições submáximas prévias para a familiarização com o procedimento de avaliação. Para a avaliação, o atleta foi estabilizado na cadeira com dois cintos no seu tronco, um cinto na pelve e outro na coxa, para evitar compensações. Uma vez o atleta posicionado da forma adequada, foi realizado o movimento de extensão e flexão de joelho da perna avaliada em uma taxa de movimento preestabelecida. Os avaliados foram incentivados verbalmente para desempenhar o máximo de força durante a execução do teste ${ }^{8}$.

Em relação aos valores ideais, assimetrias superiores a $10 \%$ entre os membros são consideradas risco elevado para lesões ${ }^{15}$. O valor de normalidade referente a relação agonista antagonista (RAA) é de $60 \%$ nas velocidades baixas $\left(60^{\circ} / \mathrm{s}-180^{\circ} / \mathrm{s}\right)^{15}$.

\section{Avaliação do controle postural}

Avaliado através da posturografia dinâmica computadorizada (PDC), Sistema EquiTest® versão 4.1. A avaliação seguiu os critérios estabelecidos pela NeuroCom, empresa fabricante do Equitest. O dispositivo Neurocom Balance Master® é padrão ouro para avaliar e estudar o equilíbrio postural estático, além de reproduzir certas situações dinâmicas das atividades físicas ${ }^{16}$. Foram avaliados os testes de organização sensorial (TOS), divididos em seis condições $^{17}$.

- condição 1: atleta em posição ortostática, plataforma fixa e olhos abertos;

- condição 2: atleta em posição ortostática, plataforma fixa e olhos fechados;

- condição 3: atleta em posição ortostática, plataforma fixa, olhos abertos e visão referenciada pelo movimento;

- condição 4: atleta em posição ortostática, plataforma em movimento e olhos abertos;

- condição 5: atleta em posição ortostática, plataforma em movimento e olhos fechados;

- condição 6: atleta em posição ortostática, plataforma e visão em movimento, com olhos abertos. 
A condição 1, 3 e 6 avalia o sistema visual, proprioceptivo e vestibular, a condição 2 e 5 avalia o sistema proprioceptivo e vestibular e a condição 4 avalia o sistema proprioceptivo ${ }^{18}$.

Baseado nos dados encontrados nestas condições, o Equitest ${ }^{\circledR}$ é capaz de calcular a média de cada uma delas, um índice de análise da função somatosensorial, visual, vestibular, assim sendo:

- função somatosensorial: média da condição 2 / média da condição 1;

- função visual: média da condição 4 / média da condição 1;

- função vestibular: média da condição 5 / média da condição 1 ;

\section{Intervenção}

O programa de prevenção de lesão utilizado no presente estudo (quadro 1) teve como base exercícios do FIFA $11+{ }^{19}$, exercícios funcionais ${ }^{20}$, e exercícios de fortalecimento de quadril $^{21}$, e ocorreu durante 12 semanas, 2 vezes por semana, por aproximadamente 40 minutos, antes do treinamento regular dos atletas.

Quadro 1 - Protocolo de prevenção de lesão

\begin{tabular}{|c|c|c|c|}
\hline EXERCÍCIOS & Nível 1 & Nível 2 & Nível 3 \\
\hline $\begin{array}{l}\text { Exercícios de } \\
\text { aquecimento }\end{array}$ & $\begin{array}{l}\text { \# Corrida para frente; } \\
\text { \& Correr joelho para fora; } \\
\text { \& Correr joelho para dentro; } \\
\text { \& Corrida à volta do parceiro; } \\
\text { \& Exercício de corrida com } \\
\text { sprints para frente e para } \\
\text { trás; } \\
\text { \# Corrida com salto e contato } \\
\text { de ombro com ombro; }\end{array}$ & $\begin{array}{l}\text { \# Corrida para frente; } \\
\text { \& Correr joelho para fora; } \\
\text { \& Correr joelho para dentro; } \\
\text { \& Corrida à volta do parceiro; } \\
\text { \& Exercício de corrida com } \\
\text { sprints para frente e para } \\
\text { trás; } \\
\text { \& Corrida com salto e contato } \\
\text { de ombro com ombro; }\end{array}$ & $\begin{array}{l}\text { \# Corrida para frente; } \\
\text { \& Correr joelho para fora; } \\
\text { \& Correr joelho para dentro; } \\
\text { \& Corrida à volta do parceiro; } \\
\text { \& Exercício de corrida com sprints } \\
\text { para frente e para trás; } \\
\text { \& Corrida com salto e contato de } \\
\text { ombro com ombro; }\end{array}$ \\
\hline $\begin{array}{l}\text { Exercícios de } \\
\text { estabilização } \\
\text { central / CORE }\end{array}$ & $\begin{array}{l}\text { \# Prancha bilateral }(3 \times 20 \mathrm{a} \\
\text { 30s) } \\
\text { \# Ponte unipodal }(2 \times 30 \mathrm{~s})\end{array}$ & $\begin{array}{l}\text { * Prancha unipodal (3x20 a } \\
\text { 30s) } \\
\text { * Ponte unipodal (2x1min) }\end{array}$ & $\begin{array}{l}\text { * Flexão funcional com faixa } \\
\text { elástica em MMSS } \\
\text { * Ponte unipodal (2x1min) }\end{array}$ \\
\hline $\begin{array}{l}\text { Exercícios de } \\
\text { agachamento }\end{array}$ & $\begin{array}{l}\text { \# Agachamento afundo } \\
(2 \times 10)\end{array}$ & $\begin{array}{l}\text { \# Agachamento com faixa } \\
\text { elástica e deslocamento } \\
\text { lateral }(2 \times 10)\end{array}$ & \\
\hline $\begin{array}{l}\text { Exercícios para } \\
\text { fortalecimento } \\
\text { do complexo } \\
\text { póstero-lateral } \\
\text { do quadril }\end{array}$ & $\begin{array}{l}\text { * Abdução de MMII em } 4 \\
\text { apoios }(2 \times 10)\end{array}$ & $\begin{array}{l}\text { * Prancha lateral dinâmica } \\
\text { joelhos fletidos }(2 \times 10)\end{array}$ & $\begin{array}{l}\text { * Prancha lateral dinâmica joelhos } \\
\text { estendidos ( } 2 \times 10) \\
\text { \# Abdução em decúbito lateral } \\
\text { com faixa elástica }(2 \times 10)\end{array}$ \\
\hline $\begin{array}{l}\text { Exercícios para } \\
\text { fortalecimento } \\
\text { de flexores da } \\
\text { coxa }\end{array}$ & \# Nórdico $(2 \times 5)$ & — Nórdico $(2 \times 5)$ & — Nórdico $(2 \times 10)$ \\
\hline $\begin{array}{l}\text { Exercícios de } \\
\text { equilíbrio }\end{array}$ & $\begin{array}{l}\text { \# Passando a bola debaixo da } \\
\text { perna }(2 \times 30 s)-\text { olhos } \\
\text { abertos; }\end{array}$ & $\begin{array}{l}\text { \# Passando a bola para o } \\
\text { colega }(2 \times 30 s)-\text { olhos } \\
\text { abertos; }\end{array}$ & $\begin{array}{l}\text { \# Elevando a bola acima da cabeça } \\
\text { (2x1min) - olhos abertos; }\end{array}$ \\
\hline
\end{tabular}




\begin{tabular}{|c|c|c|c|}
\hline & & $\begin{array}{l}\text { \# Em apoio unipodal (2x30s) - } \\
\text { olhos fechados }\end{array}$ & $\begin{array}{l}\text { \# Em apoio unipodal passando a } \\
\text { bola entre as pernas e o tronco } \\
(2 \times 30 s)-\text { olhos fechados }\end{array}$ \\
\hline $\begin{array}{l}\text { Exercícios } \\
\text { pliométricos }\end{array}$ & $\begin{array}{l}\text { \# Saltos alternados com cone } \\
(2 \times 30 \mathrm{~s})\end{array}$ & $\begin{array}{l}\text { \# Saltos alternados com cone } \\
(2 \times 30 \mathrm{~s})\end{array}$ & $\begin{array}{l}\text { \# Saltos nos cones com arremesso } \\
\text { final }(3 \times 5)\end{array}$ \\
\hline
\end{tabular}

\section{Análise estatística}

Para a análise dos dados, foi utilizado o programa SPSS, versão 20.0, no qual foi utilizada análise descritiva, por meio de medidas de média e desvio padrão. Após teste de normalidade dos dados, indicou-se uma distribuição paramétrica. Desta forma, as diferenças entre as testagens (pré e pós-testes) foram avaliadas pelo teste t de student pareado. Para todas as análises foi considerado um nível de significância de 0,05 .

\section{Resultados}

O desempenho isocinético no modo concêntrico é visualizado na tabela 2, no qual houve aumento da potência (POT) de flexores no lado dominante, e aumento no pico de torque (PT) de extensores e flexores, e na POT de extensores e flexores do lado não-dominante.

Tabela 2 - Desempenho funcional dos músculos do joelho na velocidade $60^{\circ} . \mathrm{s}-1$ do modo concêntrico pré e pós-intervenção

\begin{tabular}{lllllll}
\hline & \multicolumn{1}{c}{ PRE } & \multicolumn{1}{c}{ PÓS } & P & \multicolumn{1}{c}{ PRE } & PÓS & P \\
\hline & Dominante & Dominante & & Não Dominante & Não Dominante \\
PT extensores & $181,85 \pm 29,33$ & $192,47 \pm 27,75$ & 0,31 & $174,46 \pm 39,93$ & $205,31 \pm 30,52$ & $0,02^{*}$ \\
PT flexores & $97,75 \pm 22,55$ & $112,96 \pm 29,09$ & 0,08 & $94,47 \pm 28,68$ & $118,96 \pm 36,58$ & $0,02^{*}$ \\
TT extensores & $850,61 \pm 154,73$ & $808,81 \pm 204,57$ & 0,62 & $820,34 \pm 203,46$ & $908,27 \pm 151,08$ & 0,17 \\
TT flexores & $456,07 \pm 97,81$ & $520,42 \pm 209,59$ & 0,31 & $463,58 \pm 153,45$ & $585,77 \pm 174,10$ & 0,06 \\
POT extensores & $115,13 \pm 24,58$ & $126,16 \pm 21,78$ & 0,32 & $109,66 \pm 28,37$ & $139,86 \pm 17,33$ & $0,004^{*}$ \\
POT flexores & $61,93 \pm 17,34$ & $81,27 \pm 22,83$ & $0,03 *$ & $62,18 \pm 21,57$ & $82,73 \pm 15,30$ & $0,005^{*}$ \\
RAA & $53,47 \pm 7,01$ & $58,27 \pm 8,23$ & 0,10 & $53,67 \pm 7,84$ & $57,20 \pm 10,90$ & 0,42 \\
\hline
\end{tabular}

$\mathrm{PT}=$ pico de torque $(\mathrm{Nm}), \mathrm{TT}=$ trabalho total $(\mathrm{J}), \mathrm{POT}=$ potência (watts), RAA= relação agonista/antagonista (\%) entre flexores e extensores. Dados expressos em média e desvio padrão $\mathrm{DP} \pm$, * indica diferença significativa $(\mathrm{p}<0,05)$.

O comportamento das variáveis isocinéticas avaliadas no modo excêntrico está presente na tabela 3, no qual verificou-se aumento da POT de flexores do membro dominante. 
Tabela 3 - Desempenho funcional dos músculos do joelho na velocidade $60^{\circ} . s-1$ do modo excêntrico pré e pós-intervenção

\begin{tabular}{lllllll}
\hline & \multicolumn{1}{c}{ PRE } & PÓS & P & \multicolumn{1}{c}{ PRE } & PÓS & P \\
\hline \multirow{2}{*}{ PT extensores } & Dominante & Dominante & & Não Dominante & Não Dominante & \\
PT flexores & $141,61 \pm 22,92$ & $174,46 \pm 102,29$ & 0,42 & $124,11 \pm 34,48$ & $135,53 \pm 23,22$ & 0,24 \\
TT extensores & $445,90 \pm 146,95$ & $519,32 \pm 135,51$ & 0,36 & $445,65 \pm 184,90$ & $455,42 \pm 70,55$ & 0,88 \\
TT flexores & $891,32 \pm 206,21$ & $753,32 \pm 101,05$ & 0,21 & $794,80 \pm 192,90$ & $770,13 \pm 126,41$ & 0,72 \\
POT extensores & $74,40 \pm 17,48$ & $81,75 \pm 14,12$ & 0,14 & $70,81 \pm 20,83$ & $77,98 \pm 13,98$ & 0,17 \\
POT flexores & $64,96 \pm 13,96$ & $86,93 \pm 15,55$ & $0,005 *$ & $78,23 \pm 14,16$ & $85,47 \pm 24,01$ & 0,27 \\
RAA & $67,85 \pm 8,84$ & $64,61 \pm 5,47$ & 0,15 & $64,57 \pm 7,00$ & $67,57 \pm 8,66$ & 0,22
\end{tabular}

$\mathrm{PT}=$ pico de torque $(\mathrm{Nm}), \mathrm{TT}=$ trabalho total $(\mathrm{J}), \mathrm{POT}=$ potência (watts), RAA= relação agonista/antagonista (\%) entre flexores e extensores. Dados expressos em média e desvio padrão $\mathrm{DP} \pm,{ }^{*}$ indica diferença significativa $(\mathrm{p}<0,05)$.

O controle postural dos atletas antes e após a intervenção está descrito na tabela 4. Quanto às condições dos TOS, foi possível verificar um aumento das condições V e VI pósintervenção, além do valor de composite. Quanto à análise dos sistemas sensoriais, o sistema vestibular aumentou significativamente após a intervenção.

Tabela 4 - Controle postural das atletas pré e pós-intervenção

\begin{tabular}{llll}
\hline Variável & PRE & POS & '? \\
$\begin{array}{l}\text { Teste de } \\
\text { Sensorial }\end{array}$ & Organização & & 0,48 \\
TOS I & $94,24 \pm 0,76$ & $94,62 \pm 1,57$ & 0,15 \\
TOS II & $91,54 \pm 2,51$ & $93,45 \pm 1,62$ & 0,40 \\
TOS III & $91,78 \pm 2,33$ & $90,33 \pm 3,84$ & 0,06 \\
TOS IV & $84,83 \pm 4,19$ & $89,45 \pm 3,14$ & $0,01^{*}$ \\
TOS V & $52,91 \pm 12,86$ & $68,37 \pm 8,39$ & $0,03^{*}$ \\
TOS VI & $60,12 \pm 14,60$ & $71,62 \pm 15,84$ & $0,007^{*}$ \\
Composite & $75,37 \pm 5,70$ & $81,87 \pm 4,61$ & \\
& & & 0,12 \\
Sistema Sensorial & & $0,98 \pm 0,11$ & 0,18 \\
Sistema somatossensorial & $0,96 \pm 0,02$ & $0,92 \pm 0,03$ & $0,01^{*}$ \\
Sistema visual & $0,89 \pm 0,04$ & $0,71 \pm 0,08$ & \\
Sistema vestibular & $0,55 \pm 0,13$ & & \\
\hline
\end{tabular}

TOS = Teste de Organização sensorial; dados expressos em média e desvio padrão $\mathrm{DP} \pm$, * indica diferença significativa $(\mathrm{p}<0,05)$. 


\section{Discussão}

O presente estudo demonstrou que a prática de 12 semanas de um programa de prevenção de lesão melhorou algumas variáveis do controle postural e do desempenho funcional de joelho em atletas jovens de basquetebol masculino.

A preocupação com a prevenção de lesões em membros inferiores de atletas de basquetebol tem sido extensamente discutida na literatura ${ }^{5,22}$. Nesse contexto, uma metanálise ${ }^{23}$ incluindo 10 estudos, analisou a eficácia dos atuais programas de prevenção de lesões em membros inferiores em atletas de basquetebol. Como resultado, esses programas reduziram significativamente a incidência de lesões gerais em membros inferiores nos atletas. Um estudo randomizado realizado por Longo ${ }^{24}$, analisou, durante 9 meses, um trabalho preventivo com 11 equipes de basquetebol. Os resultados mostraram que essa abordagem, baseada no programa FIFA 11+, foi efetiva na redução das taxas de lesões em jogadores de basquete masculinos de elite. Sendo assim, sugere-se que as melhoras obtidas pelo presente estudo possam reduzir o risco de novas lesões musculoesqueléticas nos atletas avaliados.

Em relação aos dados de performance funcional do joelho, percebemos, em nosso estudo, ganhos no PT e POT dos flexores da coxa no modo concêntrico, bem como, na POT de flexores da coxa no modo excêntrico. Esses ganhos são fundamentais no ponto de vista de prevenção de lesão, uma vez que Timmins ${ }^{25}$ encontrou que baixos níveis de força muscular excêntrica de flexores da coxa aumentou o risco de lesão muscular em jogadores de futebol de elite. Ainda complementa que, como essa musculatura é exigida excentricamente durante a fase de balanço terminal da marcha, ou seja, desacelera o membro na corrida, níveis baixos de força excêntrica podem reduzir a capacidade dos isquiotibiais de fazê-lo e, como resultado, potencialmente, pode levar a uma lesão aguda. Em analogia, o jogador de basquete, assim como o de futebol, também realiza movimentos de aceleração e desaceleração bruscas durante o jogo, necessitando de um alto controle excêntrico dos flexores da coxa; o que torna o resultado obtido pelo presente estudo importante no contexto preventivo dessa modalidade.

Além do aspecto de prevenção de lesão, cabe destacar que as variáveis isocinéticas também proporcionam dados importantes no que tange à performance esportiva. Segundo Fonseca et al. ${ }^{26}$, déficits na POT, avaliados em velocidade angular baixa, poderiam influenciar o desempenho no movimento de arrancada durante uma partida, e, portanto, esse fator deve ser corrigido nos treinamentos, a fim de evitar queda de rendimento. Uma vez que houve ganhos 
nessa variável pós-intervenção, é possível que o programa utilizado também possa ter contribuído sobre a performance dos atletas desse estudo.

Adicionalmente, acreditamos que as melhorias obtidas tenham ocorrido devido às próprias características do protocolo realizado, que incluiu exercícios para controle excêntrico dos flexores da coxa (exercícios nórdicos), exercícios de ponte e pliométricos. Nesse ponto, Asadi $^{27}$ destaca que os exercícios pliométricos como os saltos aumentam o controle postural e reduzem as taxas futuras de lesões em extremidades inferiores.

Considerando que as formas de intervenção que permitam replicar o ato esportivo com maior semelhança possível ao jogo possuem maior taxa de sucesso, inserimos o treinamento pliométrico de saltos associados aos arremessos de basquete, simulando um gesto motor típico de jogo. Esse aspecto é importante, uma vez que, habitualmente, os protocolos para prevenção de lesão em atletas de basquetebol encontrados na literatura são oriundos de outros esportes, especialmente o futebol. Apesar de a grande maioria ter eficácia comprovada, é necessário à implementação de um programa específico para atender as exigências da atividade desportiva ${ }^{23}$.

Nesse estudo, apesar de não haver melhora significativa sobre a RAA, cabe destacar que os valores ficaram mais próximos da normalidade $(60 \%)^{15}$ tanto no membro dominante $(53,47 \pm 7,01$ vs. $58,27 \pm 8,23)$, quanto no não-dominante $(53,67 \pm 7,84$ vs. $57,20 \pm 10,90)$ pósintervenção. De forma semelhante, Soares et al. ${ }^{28}$ encontraram, em quatorze jogadores de futsal masculino (12,58 $\pm 0,66$ anos), resultados importantes sobre a RAA, tanto do membro dominante $(54,51 \pm 4,29$ vs $57,98 \pm 4,38)$, quanto do membro não-dominante $(49,96 \pm 8,40$ vs $52,30 \pm 6,62)$, após 18 semanas de prática com o programa FIFA11+, indicando que esse protocolo contribuiu para reduzir as assimetrias unilaterais.

Em relação aos dados de controle postural, obtivemos ganhos nas condições V (que avalia os sistemas proprioceptivo e vestibular) e VI (que avalia os três sistemas sensoriais) dos TOS, o valor de composite, além do sistema vestibular, quando analisado separadamente, dos atletas pós-intervenção. Corroborando, o estudo de Escobar et al. ${ }^{14}$ encontrou melhorias sobre o controle postural de nove atletas de basquetebol masculino adulto, após a prática de 12 semanas de um programa de prevenção de lesão baseado no protocolo FIFA11+. Esses autores encontraram um aumento da condição VI do TOS e do valor de composite pós-intervenção, avaliado pela PDC, semelhantemente ao nosso estudo.

Cabe destacar que a melhora do controle postural pode estar associada a redução de risco de lesão, conforme evidencia a metanálise proposta por Emery ${ }^{29}$. Esse autor encontrou, através de uma análise de 25 estudos randomizados e controlados, que os exercícios de 
treinamento neuromuscular (equilíbrio, agilidade e força) são benéficos e possuem um efeito protetor sobre os membros inferiores de atletas em equipes juvenis de esportes coletivos. Adicionalmente, Riva $^{30}$ demonstrou, em um estudo prospectivo de seis anos, a eficácia de programas de treinamentos proprioceptivos sobre a incidência de lesões em membros inferiores e coluna lombar em atletas de basquetebol. Nesses atletas, avaliados durante três temporadas, o treinamento preventivo demonstrou melhora no controle postural e redução dos índices de lesões nesses jogadores.

Como limitações do estudo, destaca-se: a lacuna de estudos sobre protocolos de prevenção em atletas de basquetebol de categorias de base; a baixa adesão aos treinos preventivos pelos atletas e a ausência de um grupo controle, para permitir comparações mais conclusivas.

\section{Conclusão}

O presente estudo demonstrou efeitos benéficos de um protocolo de prevenção de lesão sobre o controle postural e a performance muscular de membros inferiores de jovens atletas de basquetebol. Os ganhos sobre esses fatores são considerados imprescindíveis para a prevenção de lesão desses atletas.

\section{Referências}

1. Moreira A, Souza M, Oliveira PR. A velocidade de deslocamento no basquetebol. Rev. Bras. Ciênc. Esporte. 2003; 24(2): 201-215.

2. Fried D, Samuel C. The plasma dispersion function: the Hilbert transform of the Gaussian. Academic Press, 2015.

3. Moreira P, Gentil D, Oliveira C. Prevalência de lesões na temporada 2002 da Seleção Brasileira Masculina de Basquete. Rev. Bras. Med. Esporte. 2003; 9(5): 258-262.

4. Zuckerman SL, Wegner AM, Roos KG, Djoko A, Dompier TP, Kerr ZY. Injuries sustained in National Collegiate Athletic Association men's and women's basketball. 2009/20102014/2015. Br J Sports Med. 2018;52(4):261-268.

5. Leppänen M, Pasanen K, Kannus P, Vasankari T, Kujala UM, Heinonen A4, Parkkari J. Epidemiology of Overuse Injuries in Youth Team Sports: A 3-year Prospective Study. Int J Sports Med. 2017;38(11):847-856. 
6. Masuda K, Kikuhara N, Demura S, Katsuta S, Yamanaka K. Relationship between muscle strength in various isokinetic movements and kick performance among soccer players. J Sports Med Phys Fitness. 2005;45(1):44-52.

7. Newman MA, Tarpenning KM, Marino FE. Relationships between isokinetic knee strength, single-spring performance, and repeated-sprint ability in football players. J Strength Cond Res. 2004;18:867-72.

8. Dvir Z. Equipamento, parâmetros de teste e resultados em testes. In: Dvir Z, editor. Isocinética Avaliações musculares, interpretações e aplicações clínicas. São Paulo: Manole; 2002.

9. Terreri ASAP, Greve JMD, Amatuzzi MM. Avaliação isocinética no joelho do atleta. Rev. Bras. Med. Esporte. 2001;7(2): 62-6.

10. Freire TR, Santana MMS, Farias Neto JP. Análise do desempenho físico e do equilíbrio sob influência da crioterapia em atletas de futsal. Rev. Bras. Med. Esporte. 2015;21(6):480484.

11. Andersson E, Sward L, Thorstensson A. Trunk muscle strength in athletes. Med Sci Sports Exerc. 1988;20(6):587-93.

12. Holm I, Vollestad N. Significant effect of gender on hamstring-to-quadriceps strength ratio and static balance in prepubescent children from 7 to 12 years of age. Am J Sports Med. 2008;36(10):2007-2013.

13. Silvestre MV, Lima WC. Importância do treinamento proprioceptivo na reabilitação de entorse de tornozelo. Fisioter Mov. 2003;16(2):27-34.

14. Escobar AAJA, Lara S, Azevedo RR, Castro AAA de, Balk R de S. Benefícios do treinamento funcional em conjunto com o FIFA 11+ no controle postural de atletas de basquetebol. Rev Bras Ciên Esporte. 2019;41(1):73-80.

15. Zabka, Furlan F, Valente HG, Pacheco AM. Avaliação isocinética dos músculos extensores e flexores de joelho em jogadores de futebol profissional. Rev. Bras. Med. Esporte. 2011;17(3):189-192.

16. Ben S, Missaoui B, Miri I, Ben Salah FZ, Dziri C. Role of the Neurocom Balance Master in assessment of gait problems and risk of falling in elderly people. In: Annales de readaptation et de medecine physique: revue scientifique de la Societe francaise de reeducation fonctionnelle de readaptation et de medecine physique. 2006;49(5):210-217.

17. Medeiros IRT, Bittar RSM, Pedalini MEB, Lorenzi MC, Kii MA, Formigoni LG. Avaliação do tratamento dos distúrbios vestibulares na criança através da posturografia dinâmica computadorizada: resultados preliminares. J. Pediatr. 2003; 79(4):337-342.

18. Castagno LA. A new method for sensory organization tests: the foam-laser dynamic posturography. Braz. j. otorhinolaryngol. 1994; 60(4):287-96. 
19. Soligard T, Myklebust G, Steffen K, Holme I, Silvers H, Bizzini M, Et al. Comprehensive warm-up programme to prevent injuries in young female footballers: cluster randomised controlled trial. BMJ. 2008;337.

20. Evangelista AL, Monteiro AG. Treinamento Funcional - Uma Abordagem Pratica 2ed. São Paulo: Phorte Editora; 2012.

21. Selkowitz, DM, Beneck, GJ, Powers CM. Which Exercises Target the Gluteal Muscles While Minimizing Activation of the Tensor Fascia Lata? Electromyographic Assessment Using Fine-Wire Electrodes. J Orthop Sports Phys Ther. 2013;43(2):54-64.

22. Padua E, D'Amico AG, Alashram A, Campoli F, Romagnoli C, Lombardo M, Quarantelli M, Di Pinti E, Tonanzi C, Annino G. Effectiveness of Warm-Up Routine on the Ankle Injuries Prevention in Young Female Basketball Players: A Randomized Controlled Trial. Medicina (Kaunas). 2019;16:55(10). pii: E690.

23. Taylor, Jeffrey B., et al. Prevention of lower extremity injuries in basketball: a systematic review and meta-analysis. Sports health. 2015;7(5):392-398.

24. Longo UG, Loppini M, Berton A, Marinozzi A, Maffulli N, Denaro V. The FIFA 11+ Program Is Effective in Preventing Injuries in Elite Male Basketball Players A Cluster Randomized Controlled Trial. Am J Sports Med. 2012;40(5):996-1005.

25. Timmins RG, Bourne MN, Shield AJ, Williams MD, Lorenzen C, Opar DA. Short biceps femoris fascicles and eccentric knee flexor weakness increase the risk of hamstring injury in elite football (soccer): a prospective cohort study. Br J Sports Med. 2016;50(24):1524-1535.

26. Fonseca ST. da, Ocarino JM, Silva PLP. da, Bricio RS, Costa, CA, Wanner, LL. Caracterização da performance muscular em atletas profissionais de futebol. Rev. Bras. Med. Esporte. 2007;13(3):143-147.

27. Asadi A, Saez de Villarreal E, Arazi H. The effects of plyometric type neuromuscular training on postural control performance of male team basketball players. J Strength Cond Res. 2015; 29(7): 1870-1875.

28. Soares TS dos S, Teixeira LP, Lara S. Desempenho isocinético de atletas de futsal sub-13 após a prática do protocolo Fifa 11+. Fisioter Pesqui. 2019;26(1):1-7.

29. Emery CA, Roy TO, Whittaker JL, Nettel-Aguirre A, van Mechelen W. Neuromuscular training injury prevention strategies in youth sport: a systematic review and meta-analysis. $\mathrm{Br}$ J Sports Med. 2015;49(13):865-870.

30. Riva D, Bianchi R, Rocca F, Mamo C. Proprioceptive training and injury prevention in a professional men's basketball team: a six-year prospective study. J Strength Cond Res. 2016;30(2):461-475. 